Ruben Chavez Ayala'

Leonor Rivera-Rivera'

Angélica Angeles-Llerenas ${ }^{1}$

Eva Díaz-Cerón"

Betania Allen-Leigh'

Eduardo Lazcano Ponce

Instituto Nacional de Salud Pública. Cuernavaca, Morelos, México

Hospital 5 de Diciembre. Instituto de Seguridad y Servicios Sociales de los Trabajadores del Estado. Mexicali, Baja California, México

Correspondencia | Correspondence: Leonor Rivera Rivera

Universidad $\# 655$

Sta. Ma. Ahuacatitlán

CP 62508

Cuernavaca, Morelos, México

E-mail: Irivera@insp.mx

Recibido: 18/04/2008

Revisado: 01/10/2008

Aprobado: 12/10/2008

\section{Factores del abuso sexual en la niñez y la adolescencia en estudiantes de Morelos, México}

\author{
Factors for sexual abuse during \\ childhood and adolescence in \\ students of Morelos, Mexico
}

\section{RESUMEN}

OBJETIVO: Estimar la prevalencia y los factores asociados al abuso sexual en niñez y adolescencia.

MÉTODOS: Estudio realizado en una muestra de estudiantes del estado de Morelos, México, en 2004-2005. Los participantes $(\mathrm{n}=1730)$ pertenecen a una cohorte de 13.293 estudiantes de 12 a 24 años. Los datos fueron colectados mediante la aplicación de un cuestionario conteniendo secciones de escalas validadas. Las variables analizadas fueron: factores sociodemográficos (sexo, zona de habitación, nivel socioeconómico); familiares (educación de los padres, adicciones de los padres, violencia entre padres); psicológicos individuales (autoestima - Inventario de Autoestima de Coopersmith, depresión, consumo de alcohol); violencia intrafamiliar (Escala de Strauss); y abuso sexual. Mediante regresión logística múltiple se evaluaron los factores asociados. Se obtuvieron Razones de Momios (RM) con intervalos de confianza al 95\%.

RESULTADOS: El 4.7\% $(\mathrm{n}=80)$ de los (as) estudiantes presentaron intento de abuso y el $2.9 \%(\mathrm{n}=50)$ fueron víctimas de abuso sexual consumado. Las mujeres tuvieron mayor prevalencia de intento (6.1\%). El 3.6\% de las mujeres y el $1.9 \%$ de los hombres fueron abusados sexualmente. Principal agresor en mujeres fue el novio y en hombres una persona desconocida. Edad promedio de 12.02 años en mujeres y 11.71 en hombres. Factores asociados al abuso: mayor consumo de alcohol padres ( $\mathrm{RM}=3.37$; IC 95\% 1.40;8.07); violencia hacia madre ( $\mathrm{RM}=4.49$; IC 95\%1.54;13.10); ser mujer ( $\mathrm{RM}=2.47$; IC 95\%1.17;5.24); ser víctima de violencia intrafamiliar alta (RM=3.58; IC $95 \% 1.32 ; 9.67)$. Autoestima alta fue un factor protector ( $\mathrm{RM}=0.27$; IC $95 \%$ $0.09 ; 0.75)$.

CONCLUSIONES: En promedio el abuso sexual se presenta a los 12 años de edad en ambos sexos, siendo más frecuente en el sexo femenino. La mayoría de víctimas no lo denuncia.

DESCRIPTORES: Niño. Adolescente. Abuso Sexual Infantil. Violencia Sexual. Factores Socioeconómicos. Vulnerabilidad en Salud. Género y Salud. 


\section{ABSTRACT}

OBJECTIVE: To estimate the prevalence and factors associated with sexual abuse in childhood and adolescence.

METHODS: Study conducted in a sample of students in the state of Morelos, Mexico, in 2004-2005. Participants $(n=1730)$ were drawn from a cohort of 13,293 students aged 12 to 24 years. Data were collected by means of a questionnaire comprising parts of validated scales. The variables studied were: sociodemographic (gender, living area, socioeconomic status), family (parental education, parental addictions, violence between parents), individual psychological factors (self-esteem assessed using the Coopersmith Self-Esteem Inventory, depression, alcohol consumption), intrafamily violence (assessed through Strauss Scale) and sexual abuse. Multiple logistic regression assessed the risk factors associated. Odds ratios (OR) with $95 \%$ confidence intervals were estimated.

RESULTS: Of all students studied, $4.7 \%(\mathrm{n}=80)$ reported attempted sexual abuse and $2.9 \%(\mathrm{n}=50)$ were victims of consummated sexual abuse. Women had higher prevalence of attempted (6.1\%) abuse; $3.6 \%$ of females and $1.9 \%$ of men were sexually abused. Main perpetrators were boyfriends in women and a stranger in men. Mean age was 12.02 years old among females and 11.71 years old among men. Factors found to be associated with abuse: high parental alcohol consumption ( $\mathrm{OR}=3.37,95 \% \mathrm{CI} 1.40 ; 8.07)$, violence toward the mother (OR $=4.49,95 \%$ CI $1.54 ; 13.10)$, female gender $(\mathrm{OR}=2.47,95 \% \mathrm{CI} 1.17 ; 5.24)$, being a victim of great domestic violence $(\mathrm{OR}=3.58,95 \% \mathrm{CI} 1.32 ; 9.67)$. High self-esteem was a protective factor ( $\mathrm{OR}=0.27,95 \% \mathrm{CI} 0.09 ; 0.75)$.

CONCLUSIONS: Overall sexual abuse occurs at the age of 12 in both males and females, and it is more frequent among females. Most victims do not report abuse.

DESCRIPTORS: Child. Adolescent. Child Abuse, Sexual. Sexual Violence. Socioeconomic Factors. Health Vulnerability. Gender and Health.

\section{INTRODUCCIÓN}

El abuso sexual en la infancia es reconocido como un factor de riesgo asociado a trastornos mentales de tipo depresivo, de ansiedad, de personalidad, sexuales, fobia social, adicciones y padecimientos físicos como las infecciones sexuales, entre otros. ${ }^{5,21}$

En la mayoría de los casos están implicados miembros de la propia familia o conocidos por lo que los casos de abuso son mantenidos como secreto familiar y en consecuencia se transmiten generacionalmente. ${ }^{18} \mathrm{Se}$ estima que entre el $2 \%$ al $59 \%$ de los niños han sido víctimas de este problema. ${ }^{20}$

En estudios realizados en estudiantes de la Ciudad de México se encontró que el $7 \%$ de las mujeres y $2 \%$ de los hombres habían sufrido abuso sexual ${ }^{8}$ y que el promedio de edad en que ocurre es entre los 9 y 13 años. ${ }^{17}$

Entre los factores de riesgo familiares se señalan el abuso del alcohol de los padres ${ }^{9}$ y violencia hacia la madre, ${ }^{10}$ entre otros, y como factores individuales, se mencionan el hecho de ser mujer, ${ }^{6}$ haber sido víctima de violencia familiar y tener una autoestima baja.

Por lo anteriormente señalado, el objetivo del presente estudio fue estimar la prevalencia y los factores asociados al abuso sexual durante la niñez y adolescencia.

\section{MÉTODOS}

El estudio se realizó en una cohorte que incluyó a 13,293 estudiantes, entre 12 y 24 años, del Estado de Morelos (México), seleccionados aleatoriamente de 260 escuelas secundarias, 92 escuelas preparatorias y una universidad. De esta medición, solo participaron los 1.730 alumnos que aún estaban inscritos en las escuelas durante el periodo escolar 2003-2004. Primero se obtuvieron los listados de todos los alumnos que se encontraban en cada escuela y se ubicaron por grupo a todos aquéllos que habían participado en el estudio anteriormente. Se les hizo una invitación a seguir par- 
ticipando y se les entregaron cartas de consentimiento informado y de autorización para los padres, cuando el estudiante era menor de edad.

Posteriormente, ajustándonos a los horarios de los estudiantes y organizándolos en pequeños grupos dependiendo de su disponibilidad de tiempo, se realizó la encuesta entre 2004 y 2005 con un cuestionario autoaplicable. El procedimiento se llevó a cabo en cada institución hasta encuestar la totalidad de los estudiantes. El tiempo de aplicación promedio fue de 40 minutos. Un 3\% fueron encuestados en sus hogares porque se encontraban en prácticas profesionales o porque no se les pudo localizar en las aulas por diversas causas, como baja temporal o embarazo. El cuestionario que recibieron los participantes tuvo las siguientes secciones: factores sociodemográficos, familiares, psicológicos individuales, violencia intrafamiliar, y abuso sexual.

El nivel socioeconómico se construyó a partir de las siguientes variables: material del piso de la vivienda, disponibilidad de agua entubada, infraestructura para la eliminación de excretas, número de personas que habitan la vivienda, número de cuartos, y escolaridad del (o la) jefe de familia.

Entre los factores familiares, el consumo inmoderado de alcohol del padre fue determinada haciendo la siguiente pregunta: “¿Tu padre consume alcohol?”. Para el análisis del consumo de alcohol, se construyó una variable con tres categorías: $0=$ nunca (no consumo de alcohol o consumo de una vez al año), $1=$ menor consumo (consumo de alcohol de cada quince días hasta dos a cinco veces al año) y $2=$ mayor consumo (diario y cada ocho días).

La violencia hacia la madre se midió con la pregunta: 1.-¿Tu papá golpea a tu mamá?

El índice de violencia familiar se construyó con 21 reactivos de la Escala de Strauss, ${ }^{22}$ que preguntó si algún miembro de la familia (madre/madrastra, padre/padrastro, hermanos, tíos, abuelos) había cometido alguno de los actos violentos mencionados en un listado: insultos, diversas amenazas físicas, amenazas con distintos tipos de armas, golpes con objetos, jaloneo del cuerpo, golpes, quemaduras intencionales, ahorcamiento, lesiones producidas por armas, entre otros.

Se realizó un análisis factorial por el método de componentes principales con rotación varimax de esta escala, obteniéndose cuatro factores. Los factores uno y dos representaban actos de violencia no severos como insultos, jaloneos y golpes con objetos. Los factores tres y cuatro agruparon actos de violencia severos como amenazas de lesiones producidas por armas, amenaza de muerte, quemaduras, intentos de ahorcamientos y lesiones con armas. Para construir el índice de violencia familiar se consideraron los reactivos de los factores uno y dos. Para obtener una variable de violencia familiar en tres categorías, se sumaron los 11 reactivos correspondientes a los factores uno y dos y se dividió por terciles: sin violencia, violencia baja y violencia alta.

La autoestima se midió por el Inventario de Autoestima de Coopersmith. Por Análisis Factorial se obtuvo un primer factor $(\alpha=0.84)$ al que se denominó "autoestima negativa".

Para la definición de abuso sexual en menores de edad, se partió de la definición de Wyatt \& Russel1 ${ }^{19,25}$ con la limitación de desconocer la edad del agresor. Se consideró al intento de violación como un conato de abuso sexual; y por abuso sexual consumado, a la violación sexual consumada cuando ocurrió sin consentimiento en menores de 18 años. Estas variables se midieron mediante dos preguntas: “¿Alguna vez intentaron obligarte a tener relaciones sexuales?” " “¿Alguna vez te han obligado a tener relaciones sexuales en contra de tu voluntad?". Después se crearon las dos variables respuesta: intento de abuso sexual (cuando la persona respondió afirmativamente a la pregunta sobre intento y negativamente a la de abuso consumado) y abuso sexual consumado (cuando la persona respondió afirmativamente a la pregunta sobre abuso sexual consumado, independientemente de cómo haya contestado a la pregunta sobre intento).

Se realizó un análisis bivariado, con prueba de ji cuadrada, para analizar asociación entre intento de abuso sexual y abuso sexual consumado por las variables independientes. Se utilizó análisis de regresión logística múltiple para controlar las posibles variables confusoras, ajustando por nivel socioeconómico, sexo, violencia familiar, autoestima, violencia hacia las madres, consumo inmoderado de alcohol del padre. El análisis se realizó por medio del paquete estadístico de Stata versión 8.0.

El estudio siguió los principios de la Declaración de Helsinski y fue aprobado por la Comisión de Ética del Instituto Nacional de Salud Pública.

\section{RESULTADOS}

De los 1.730 estudiantes que constituyeron la muestra de este estudio, 1.045 (60.4\%) fueron mujeres; 1.658 (96.1\%) tenían entre 16 a 24 años de edad; 1.193 $(68.8 \%)$ pertenecían a los niveles socioeconómicos bajo y medio. En relación al tipo de localidad el 589 $(34.2 \%)$ fueron residentes del área rural.

En la Tabla 1, se observan las características de las variables de estudio por sexo. Se encontraron diferencias significativas en consumo de alcohol paterno, violencia hacia la madre e intrafamiliar $(\mathrm{p}<0.05)$. En relación al nivel socioeconómico se observó que la mayoría de los estudiantes de ambos sexos pertenecieron al nivel 
Tabla 1. Características de las variables de los estudiantes de escuelas públicas por sexo. Estado de Morelos, México, 20042005.

\begin{tabular}{|c|c|c|c|c|c|c|c|}
\hline \multirow{2}{*}{ Característica } & \multicolumn{2}{|c|}{ Total } & \multicolumn{2}{|c|}{ Hombres } & \multicolumn{2}{|c|}{ Mujeres } & \multirow[b]{2}{*}{$\mathrm{p}$} \\
\hline & $\mathrm{n}$ & $\%$ & $\mathrm{n}$ & $\%$ & $\mathrm{n}$ & $\%$ & \\
\hline \multicolumn{8}{|l|}{ Nivel socioeconómico } \\
\hline Bajo & 299 & 17.2 & 95 & 13.8 & 204 & 19.5 & \\
\hline Medio & 894 & 51.6 & 401 & 58.5 & 493 & 47.1 & \\
\hline Alto & 537 & 31.0 & 189 & 27.5 & 348 & 33.3 & 0.000 \\
\hline \multicolumn{8}{|l|}{ Tipo de zona } \\
\hline Rural & 589 & 34.2 & 255 & 37.2 & 334 & 31.9 & \\
\hline Suburbana & 445 & 25.7 & 162 & 23.6 & 283 & 27.0 & \\
\hline Urbana & 696 & 40.2 & 268 & 39.1 & 428 & 40.9 & 0.061 \\
\hline \multicolumn{8}{|c|}{ Consumo inmoderado de alcohol del padre } \\
\hline Nunca & 1073 & 62.0 & 396 & 57.8 & 677 & 64.7 & \\
\hline Menor consumo & 224 & 2.9 & 103 & 15.0 & 121 & 11.5 & \\
\hline Mayor consumo & 433 & 2.0 & 186 & 27.1 & 247 & 23.6 & 0.011 \\
\hline \multicolumn{8}{|c|}{ Violencia hacia la madre } \\
\hline Ausencia & 1363 & 95.6 & 549 & 96.3 & 814 & 95.2 & \\
\hline Presencia & 62 & 4.3 & 21 & 3.6 & 41 & 4.8 & 0.000 \\
\hline \multicolumn{8}{|l|}{ Autoestima } \\
\hline Baja & 509 & 29.4 & 189 & 27.5 & 320 & 30.6 & \\
\hline Media & 677 & 39.1 & 268 & 39.1 & 409 & 39.1 & \\
\hline Alta & 543 & 31.4 & 228 & 33.2 & 315 & 30.1 & 0.274 \\
\hline \multicolumn{8}{|l|}{ Violencia intrafamiliar } \\
\hline Sin violencia & 692 & 52.5 & 284 & 57.3 & 408 & 49.6 & \\
\hline Ваја & 356 & 27.0 & 120 & 24.2 & 236 & 28.7 & \\
\hline Alta & 269 & 20.4 & 91 & 18.3 & 178 & 21.6 & 0.024 \\
\hline
\end{tabular}

socioeconómico medio (hombres $58.5 \%$ y en mujeres $47.2 \%)$. En relación al tipo de zona en que viven, el $39.1 \%$ de los hombres y $41.0 \%$ de mujeres pertenecieron a la urbana.

Se encontró que el 4.7\% $(n=80)$ de los estudiantes sufrieron intento de abuso sexual y el $2.9 \%(n=50)$ fueron víctimas de abuso sexual consumado.

Las mujeres presentaron una mayor prevalencia de intento de abuso sexual en comparación con los hombres (6.1\% y $2.6 \%$, respectivamente). Al comparar el abuso sexual consumado por sexo, el 3.6\% de las mujeres fueron víctimas en comparación con $1.9 \%$ de los hombres.

Por promedio de edad en que ocurrieron tanto el intento de abuso como el abuso sexual consumado se encontró que no existe diferencia significativa por sexo. En el caso de las mujeres, el promedio de edad en que experimentaron intento de abuso sexual fue de 11.85 años (ds 5.19); y en los hombres fue 10.66 años (ds 4.50). Respecto a la edad del abuso sexual consumado, en las mujeres ocurrió a los 12.02 años (ds 6.10) y en hombres a los 11.71 años (ds 5.60).
En la Figura 1, se describe a los agresores de intento de abuso sexual. En las mujeres el principal agresor fue un desconocido $(30.5 \%)$, seguido por otras personas no especificadas (20.2\%), el novio (19.6\%), el vecino y el tío ( $13.7 \%$, respectivamente) y el padre o padrastro y hermano (3.2\%, respectivamente). Cuando la víctima es masculina, el principal agresor fue un desconocido $(37.0 \%)$, siguiendo en orden de frecuencia: vecinos (25\%), otros agresores no especificados (18.5\%), novia y tío (11.1\%, respectivamente), tía (3.7\%) y padre o padrastro $(2.4 \%)$. En ambos sexos hubo casos de revictimización en intento de abuso sexual. En las mujeres el porcentaje fue de $11.5 \%$ y en los hombres fue de $14.3 \%$.

En la Figura 2, se observa el tipo de agresor de abuso sexual consumado por sexo. En las mujeres el principal agresor fue el novio (37.8\%) seguido de otros sujetos $(30.3 \%)$, entre los cuales fueron señalados el sacerdote, el primo y el maestro; seguidos de una persona desconocida (16.2\%), el tío (13.5\%), vecino (5.4\%) y hermano, padre o padrastro $(2.7 \%$, respectivamente). En los hombres el principal agresor de abuso sexual consumado fue un desconocido (38.5\%), seguido de la 


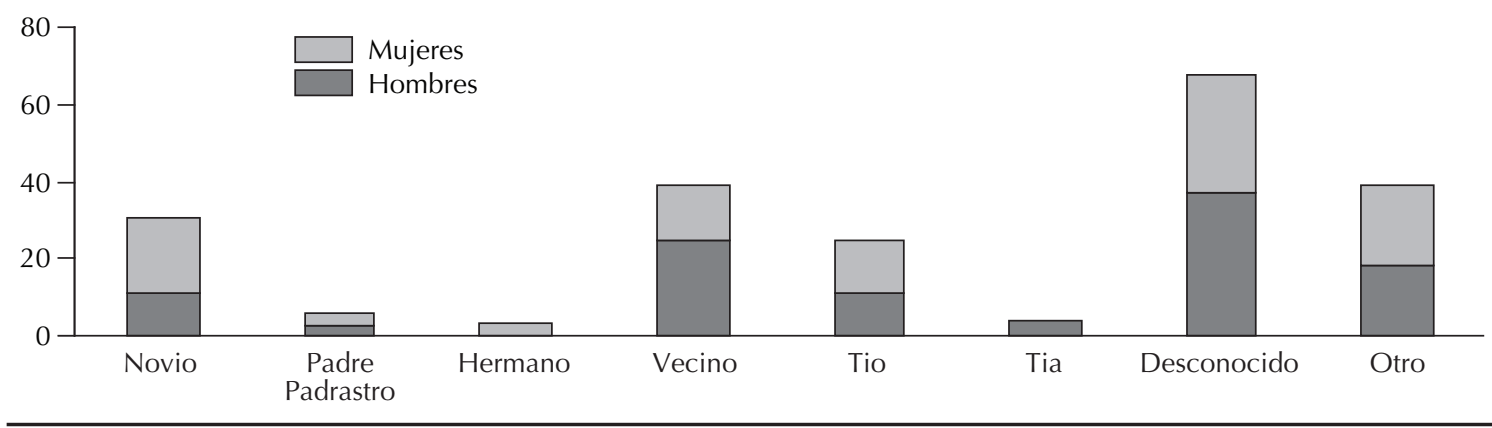

Figura 1. Tipo de agresor del intento de abuso sexual por sexo de los estudiantes de escuelas públicas. Estado de Morelos, México, 2004-2005.

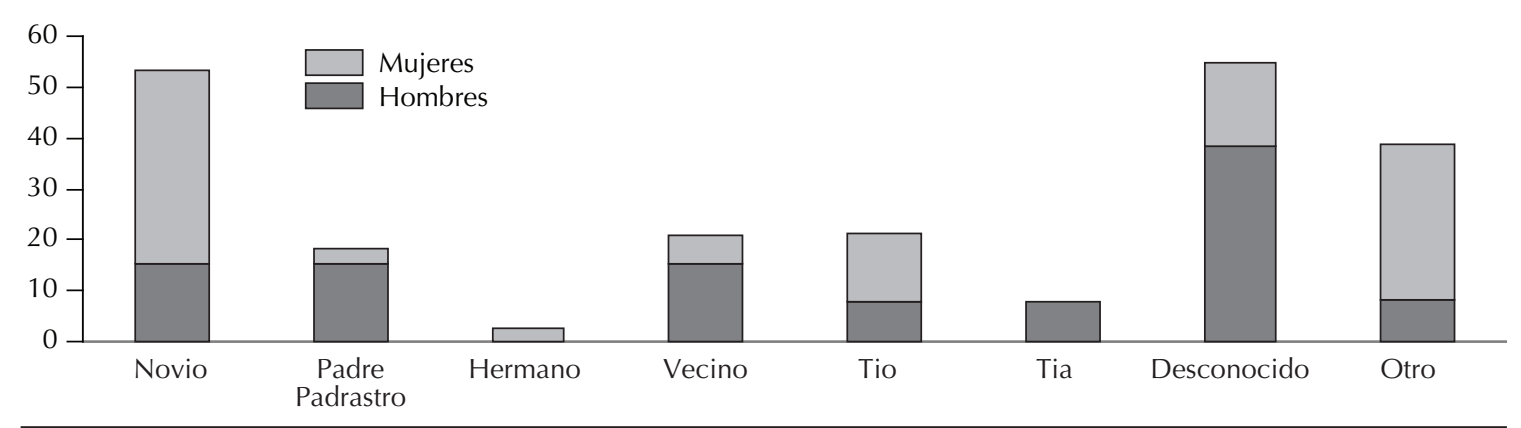

Figura 2. Tipo de agresor del abuso sexual consumado por sexo en estudiantes de escuelas públicas. Estado de Morelos, México, 2004-2005.

novia y algún vecino (15.4\%, respectivamente), el padre o padrastro $(15.3 \%)$, otros no especificados $(8.3 \%)$ y tío o tía $(7.7 \%$, respectivamente). El abuso sexual por parte de padres y padrastros fue mayor en hombres (15.4\%) que en las mujeres (2.7\%). A su vez el padre por si solo, no fue violador sexual en algún caso en las mujeres, pero si fue así con el tío (13.5\%) y el hermano (2.7\%). Entre las figuras femeninas del hogar (madre, hermana y tías) se encontró solamente a la tía como una persona abusadora tanto en el intento (3.7\%) como en el hecho consumado (7.7\%), y exclusivamente contra el menor masculino.

Cuando se les preguntó acerca de haber comentado a alguien el abuso sexual experimentado, sólo el 32.7\% lo comentaron, de los cuales el $70.6 \%$ eran mujeres. Un total de $15.4 \%$ solicitaron ayuda (siendo $62.5 \%$ mujeres). Solo el $1.9 \%$ de las víctimas de abuso consumado presentaron denuncia legal tratándose de un caso de sexo masculino y a la edad de 14 años de edad.

En la Tabla 2, se observa la prevalencia de abuso sexual con respecto a las diferentes variables de estudio. Se encontraron diferencias significativas entre la prevalencia de abuso sexual y violencia hacia la madre e intrafamiliar, sexo y autoestima $(\mathrm{p}<0.05)$.

En la Tabla 3, se observan los resultados del análisis de regresión logística múltiple, ajustado por nivel socioeconómico, consumo de alcohol del padre, violencia hacia la madre, sexo, autoestima y violencia intrafamiliar.

Los factores familiares asociados al intento de abuso sexual y al abuso sexual consumado fueron consumo de alcohol del padre y violencia hacia la madre. Los factores individuales asociados al abuso sexual consumado fueron sexo, autoestima y violencia intrafamiliar.

Los estudiantes con autoestima media y alta presentaron menor posibilidad de ser víctimas de abuso sexual en comparación con los de autoestima baja.

En relación a la variable de violencia intrafamiliar, en la Tabla 3 se observa que los estudiantes víctimas de violencia intrafamiliar baja tuvieron mayor posibilidad de presentar intento de abuso sexual y abuso sexual consumado en comparación con aquellas(os) que no presentaban violencia intrafamiliar. Ésta posibilidad se incrementa cuando las(os) estudiantes presentaban violencia familiar alta.

\section{DISCUSIÓN}

Los resultados del presente estudio señalan una prevalencia importante de casos de intento y abuso sexual consumado en estudiantes durante su minoría de edad. Las prevalencias encontradas son similares a la repor- 
Tabla 2. Prevalencia de abuso sexual en estudiantes de escuelas públicas por diferentes factores. Estado de Morelos, México, 2004-2005.

\begin{tabular}{|c|c|c|c|c|c|c|c|c|c|}
\hline \multirow{2}{*}{ Variable } & \multicolumn{2}{|c|}{ Ausencia } & \multicolumn{2}{|c|}{$\begin{array}{c}\text { Intento de abuso } \\
\text { sexual }\end{array}$} & \multicolumn{2}{|c|}{$\begin{array}{l}\text { Abuso sexual } \\
\text { consumado }\end{array}$} & \multirow{2}{*}{$\begin{array}{c}\text { Total } \\
\mathrm{n}\end{array}$} & \multirow[b]{2}{*}{$\%$} & \multirow[b]{2}{*}{$\mathrm{p}$} \\
\hline & $\mathrm{n}$ & $\%$ & $\mathrm{n}$ & $\%$ & $\mathrm{n}$ & $\%$ & & & \\
\hline \multicolumn{10}{|l|}{ Factores sociodemográficos } \\
\hline \multicolumn{10}{|l|}{ Tipo de zona } \\
\hline Rural & 539 & 34.4 & 23 & 28.7 & 17 & 34.0 & 579 & 34.2 & \\
\hline Semiurbana & 396 & 25.3 & 24 & 30.0 & 10 & 20.0 & 430 & 25.4 & \\
\hline Urbana & 629 & 40.2 & 33 & 41.2 & 23 & 46.0 & 685 & 40.4 & 0.665 \\
\hline \multicolumn{10}{|l|}{ Nivel socioeconómico } \\
\hline Bajo & 269 & 17.2 & 16 & 20.0 & 5 & 10.0 & 290 & 17.1 & \\
\hline Medio & 810 & 51.7 & 38 & 47.5 & 27 & 54.0 & 875 & 51.6 & \\
\hline Alto & 485 & 31.0 & 26 & 32.5 & 18 & 36.0 & 529 & 31.2 & 0.622 \\
\hline \multicolumn{10}{|l|}{ Factores familiares } \\
\hline \multicolumn{10}{|c|}{ Consumo inmoderado de alcohol del padre } \\
\hline Nunca & 972 & 62.1 & 53 & 66.2 & 29 & 58.0 & 1054 & 62.2 & \\
\hline Menor consumo & 205 & 13.1 & 10 & 12.5 & 5 & 10.0 & 220 & 12.9 & \\
\hline Mayor consumo & 387 & 24.7 & 17 & 21.2 & 16 & 32.0 & 420 & 24.7 & 0.702 \\
\hline \multicolumn{10}{|c|}{ Violencia hacia la madre } \\
\hline Ausencia & 1263 & 96.4 & 50 & 87.7 & 31 & 79.5 & 1344 & 95.6 & \\
\hline Presencia & 46 & 3.51 & 7 & 12.2 & 8 & 20.5 & 61 & 4.3 & 0.000 \\
\hline \multicolumn{10}{|l|}{ Factores individuales } \\
\hline \multicolumn{10}{|l|}{ Sexo } \\
\hline Hombre & 647 & 41.3 & 18 & 22.5 & 13 & 26.0 & 678 & 40.0 & \\
\hline Mujeres & 917 & 58.6 & 62 & 77.5 & 37 & 74.0 & 1016 & 59.9 & 0.000 \\
\hline \multicolumn{10}{|l|}{ Autoestima } \\
\hline Baja & 441 & 28.2 & 30 & 37.5 & 23 & 46.0 & 494 & 29.2 & \\
\hline Media & 618 & 39.5 & 33 & 41.2 & 14 & 28.0 & 665 & 39.3 & \\
\hline Alta & 504 & 32.2 & 17 & 21.2 & 13 & 26.0 & 534 & 32.2 & 0.015 \\
\hline \multicolumn{10}{|l|}{ Violencia intrafamiliar } \\
\hline Sin violencia & 654 & 54.1 & 21 & 35.5 & 8 & 25.8 & 683 & 52.7 & \\
\hline Baja & 319 & 26.4 & 20 & 33.9 & 8 & 25.8 & 347 & 26.7 & \\
\hline Alta & 234 & 19.3 & 18 & 30.5 & 15 & 48.3 & 267 & 20.5 & 0.000 \\
\hline
\end{tabular}

tadas por otras investigaciones realizadas en México, como la de Ramos-Lira et $\mathrm{al}^{17}$ con una prevalencia del $4.3 \%$ de abuso sexual en estudiantes. En términos generales, al comparar los resultados del presente estudio con otros similares realizados en el mundo, se encuentra que la prevalencia de violación sexual consumada en la población de estudiantes mexicanos estudiados es menor a la de la mayoría de los estudios revisados, para ambos sexos: en estudios realizados en los Estados Unidos se han encontrado prevalencias de abuso sexual desde $7.1 \%{ }^{3}$ hasta $31 \% ;^{4}$ en Sudáfrica, $5.9 \%{ }^{11}$ en Costa Rica, $10.8 \%$ en mujeres y $6.4 \%$ en hombres, como víctimas. ${ }^{13}$

En el presente estudio se encontró que este problema fue más frecuente en las mujeres, coincidiendo con otras investigaciones. ${ }^{8}$ Por ejemplo, en una encuesta realizada en Barbados una de cada tres mujeres y uno de cada dos hombres, reportan abuso sexual. ${ }^{9}$ Estos hallazgos pudieran estar relacionados con un desequilibrio de poder en las relaciones de género, ${ }^{12}$ y con una menor denuncia masculina del abuso. La construcción de la identidad masculina se define como un alejamiento de la homosexualidad y la feminidad, de modo que denunciar que se ha sido víctima de abuso sexual es denunciar que se ha tenido una experiencia homosexual forzada. ${ }^{16}$

En relación a la edad, el rango en el que ocurrió el abuso es similar al de otros estudios en México, ${ }^{8}$ donde se encontró que el $28.4 \%$ del total de las víctimas sufrió abuso sexual antes de los diez años de edad y el $40 \%$ entre los diez y los 13 años. 
Tabla 3. Factores asociados al abuso sexual en estudiantes de escuelas públicas. Estado de Morelos, México, 2004-2005.

\begin{tabular}{|c|c|c|c|c|}
\hline \multirow{2}{*}{ Variable } & \multicolumn{2}{|c|}{ Intento de abuso } & \multicolumn{2}{|c|}{ Abuso sexual } \\
\hline & $R M^{a}$ & IC $95 \%$ & $\mathrm{RM}^{\mathrm{a}}$ & IC 95\% \\
\hline \multicolumn{5}{|l|}{ Factores familiares } \\
\hline \multicolumn{5}{|c|}{ Consumo inmoderado de alcohol del padre } \\
\hline Nunca & 1.0 & & 1.0 & \\
\hline Menor consumo & 1.14 & $0.48 ; 2.71$ & 0.9 & $0.57 ; 6.37$ \\
\hline Mayor consumo & 1.23 & $0.59 ; 2.54$ & 3.37 & $1.40 ; 8.07$ \\
\hline \multicolumn{5}{|c|}{ Violencia hacia la madre } \\
\hline Ausencia & 1.0 & & 1.0 & \\
\hline Presencia & 1.43 & $0.40 ; 5.02$ & 4.49 & $1.54 ; 13.10$ \\
\hline \multicolumn{5}{|l|}{ Factores individuales } \\
\hline \multicolumn{5}{|l|}{ Sexo } \\
\hline Hombre & 1.0 & & 1.0 & \\
\hline Mujer & 2.47 & $1.17 ; 5.24$ & 1.53 & $0.61 ; 3.81$ \\
\hline \multicolumn{5}{|l|}{ Autoestima } \\
\hline Baja & 1.0 & & 1.0 & \\
\hline Media & 0.95 & $0.47 ; 1.92$ & 0.26 & $0.09 ; 0.72$ \\
\hline Alta & 0.66 & $0.30 ; 1.49$ & 0.27 & $0.09 ; 0.75$ \\
\hline \multicolumn{5}{|l|}{ Violencia intrafamiliar } \\
\hline Ausencia & 1.0 & & 1.0 & \\
\hline Baja & 2.37 & $1.16 ; 4.82$ & 1.58 & $0.52 ; 4.72$ \\
\hline Alta & 2.42 & $1.12 ; 5.19$ & 3.58 & $1.32 ; 9.67$ \\
\hline
\end{tabular}

a Ajustado por las variables contenidas en la tabla y por nivel socioeconómico.

Al analizar el tipo de agresor, se encontró que en cuanto al intento de abuso sexual en ambos sexos, los agresores fueron personas desconocidas; sin embargo, al analizar a los que fueron víctimas de abuso sexual consumado, tanto en mujeres como en hombres, los agresores principales no pertenecen al espacio doméstico, aunque para las mujeres el principal agresor sea un conocido (el novio). En general, nuestros hallazgos difieren con los resultados de otros estudios donde los principales agresores son personas conocidas tanto en hombres como en mujeres. ${ }^{17}$

En el presente estudio, se encontraron diversos factores familiares e individuales asociados al abuso sexual. Dentro del ambiente familiar los estudiantes que tenían padres que consumen alcohol tuvieron mayor posibilidad de presentar abuso sexual. Estos resultados son consistentes con otras investigaciones ${ }^{7}$ que señalan que el riesgo de presentar abuso sexual es cinco veces más alto en adolescentes que tuvieron padres que consumen alcohol. Así mismo, en el presente estudio fue baja la perpetración de abuso sexual en el interior del espacio doméstico por parte de ambos padres, similar a otros estudios poblacionales. ${ }^{14}$

Otro de los factores del ambiente familiar asociados al abuso sexual es la violencia que se ejerce hacia la madre. En este estudio se encontró que los estudiantes con reportes de violencia contra su madre tuvieron 4.49 veces mas riesgo de ser víctimas de abuso sexual. A su vez, se encontró que la violencia intrafamiliar también incrementa la probabilidad de presentar abuso sexual dentro de la familia, lo cual es consistente con otras investigaciones. ${ }^{15}$

Por otra parte, tener una autoestima más alta resultó ser un factor protector de ser víctima de abuso sexual, lo que coincide con investigaciones realizada en varios países sobre la asociación entre la autoestima, estilos de afrontamiento y sus efectos en el estrés post traumático del abuso sexual. ${ }^{2}$ En México se ha encontrado en población infantil, que conforme los niveles de autoestima son más altos es más probable que la persona posea un estilo de afrontamiento dirigido al control directo del problema. Esta es una variable mediadora en el desarrollo de la psicopatología derivada de experiencias negativas como las del abuso sexual. ${ }^{24}$ Mediante el afrontamiento directo la víctima puede reducir el estrés ${ }^{1}$ y con ello detener el desarrollo de la patología. Los resultados encontrados sugieren la importancia de investigar e incorporar en las intervenciones para la prevención y la atención del abuso sexual infantil talleres para el desarrollo de habilidades cognitivo conductuales de control directo del problema del abuso o de su prevención en la minoría de edad. La consecuencia es el fortalecimiento de la autoestima y 
esta a su vez, refuerza el uso de este tipo de estrategias de afrontamiento.

En este estudio se encontró que solo la tercera parte de los que son abusados sexualmente le comentan a alguien sobre el suceso. De los estudiantes que presentaron abuso sexual solo un $15.4 \%$ solicitó ayuda, siendo en su gran mayoría las mujeres; sin embargo, en cuanto a la denuncia legal solo una de las víctimas la realizó tratándose de un varón y cuando el abuso ocurrió a los 14 años de edad. Según Summit, ${ }^{23}$ la no denuncia del abuso sexual está ligada a un proceso de acomodación cognitivo-emocional de la víctima que incluso pudiera llegar a ser parte de un trastorno del desarrollo de la personalidad derivado de la misma experiencia sexual abusiva. Al ser frecuente, afecta la autoimagen y autoestima, así que la víctima no denuncia el abuso porque se percibe responsable de los hechos. Además dentro del contexto familiar el abuso puede comenzar a tener la función de estabilidad de las relaciones familiares. En el presente estudio se encontraron a tíos, tías, hermanos, padres y padrastros como abusadores en el espacio familiar, donde la ruptura del secreto de las prácticas abusivas puede desencadenar una ruptura de la unidad familiar lo que implica un riesgo para la seguridad misma de la víctima al perder el apoyo emocional familiar, lo cuál es muchas veces confirmado cuando las víctimas denuncian y se enfrentan al rechazo de su propia familia por la no credibilidad.

Entre las limitaciones del presente estudio, la prevalencia de abuso sexual pudiera estar subestimada por la manera en que se midió esta variable, limitada a dos indicadores: intento de violación y violación sexual consumada. El abuso sexual presenta otras expresiones como tocamientos, expresiones verbales obscenas, exhibicionismo $\mathrm{u}$ obligar a la víctima a observar imágenes con contenido erótico, entre otros. Tampoco se midieron otras variables que pudieran ser de interés para este estudio: perfil del agresor, ausencia de madre en el hogar, daño psicológico de víctimas, personalidad de víctimas de abuso en diversas ocasiones. Otra limitación es el diseño de este estudio ya que no se puede establecer causalidad con un diseño transversal y nuestra muestra sesga los resultados hacia una población de jóvenes que posiblemente tiene mejores condiciones familiares y económicas.

En conclusión se puede señalar que el abuso sexual es un evento que se presenta desde etapas tempranas de la vida siendo el promedio de edad en que ocurre el abuso sexual a los doce años de edad. Ambos sexos son víctimas de abuso sexual en el Estado de Morelos, México, sin embargo, éste evento es mas frecuente en las niñas. Se debe de enseñar habilidades a los jóvenes para que denuncien este delito. De las características del agresor solo indagamos quién fue el agresor y en su mayoría resultó que fue un desconocido en los casos de intento de abuso. En las mujeres el novio fue uno de los principales agresores del abuso sexual consumado. El abuso sexual es un problema que permanece oculto ya que la mayoría de los que padecen no lo comentan y no piden ayuda. Podemos decir que existen ambientes familiares que propician el abuso sexual asociado al consumo de alcohol del padre que puede propiciar una conducta violenta ejercida hacia la madre. También existen otros factores propios del individuo que de alguna manera tienen su origen en conductas violentas dentro del hogar como por ejemplo, la violencia que ejercen los miembros de la familia hacia los hijos (as); sin embargo, el desarrollo de una buena autoestima en los hijos puede propiciar un recurso de autodefensa ante situaciones de abuso.

Se requiere profundizar en esta línea de investigación a nivel poblacional, en virtud de que los datos del presente estudio solo representan a un sector de la juventud que asiste a escuelas públicas del Estado de Morelos, México; sin embargo, nuestros resultados son de gran utilidad para que el Ministerio de Educación Pública en colaboración con otras instituciones, establezcan medidas de prevención, detección y disminución de abuso sexual. 


\section{REFERENCIAS}

1. Álvarez-Icaza V. La autoestima estrés y afrontamiento desde la perspectiva de niños y niñas escolares. Hologramática. 2006;3(5):59-70.

2. Cieslak R, Benight CC, Caden Lehman V. Coping self-efficacy mediates the effects of negative cognitions on posttraumatic distress. Behav Res Ther. 2008;46(7):788-98. DOI: 10.1016/j.brat.2008.03.007

3. Champion HL, Foley KL, DuRant RH, Hensberry $\mathrm{R}$, Altman D, Wolfson M. Adolescent sexual victimization, use of alcohol and other substances, and other health risk behaviors. J Adolesc Health. 2004;35(4):321-8.

4. Erickson PI, Rapkin AJ. Unwanted sexual experiences among middle and high school youth. J Adolesc Health. 1991;12(4):319-25. DOI: 10.1016/01970070(91)90007-9

5. Filipas HH, Ullman SE. Child sexual abuse, coping responses, self-blame, posttraumatic stress disorder, and adult sexual revictimization. J Interpers Violence. 2006;21(5):652-72. DOI: 10.1177/0886260506286879

6. Filkelhor D. The international epidemiology of child sexual abuse. Child Abuse Negl. 1994;18(5):409-17. DOI: 10.1016/0145-2134(94)90026-4

7. Fleming J, Mullen P, Bammer G. A study of potential risk factors for sexual abuse in childhood. Child Abuse Negl. 1997;21(1):49-58. DOI: 10.1016/S01452134(96)00126-3

8. González-Forteza C, Ramos-Lira LE, Vignau-Brambila LE, Ramírez-Villarreal C. El abuso sexual y el intento suicida asociados con el malestar depresivo y la ideación suicida de los adolescentes. Salud Mental. 2001;24(6):16-25.

9. Heise L, Pitanguy J, Germain A. Violencia contra la mujer: la carga oculta sobre la salud. Washington: OPAS; 1994.

10. Intebi I. Abuso sexual infantil en las mejores familias. Barcelona: Granica; 1998.

11. King G, Flisher AJ, Noubary F, Reece R, Marais A, Lombard C. Substance abuse and behavioral correlates of sexual assault among South African adolescents. Child Abuse Negl. 2004;28(6):683-96. DOI: 10.1016/j. chiabu.2003.12.003

12. Krug EG, Dahlberg LL, Mercy JA, ZWI AB, Lozano R. World report on violence and health. Geneva: WHO, 2002. Sexual violence; p.149-80.

13. Krugman S, Mata L, Krugman R. Sexual abuse and corporal punishment during childhood: a pilot retrospective survey of university students in Costa Rica. Pediatrics. 1992;90(1 Pt 2):157-61.
14. Lalor K. Child sexual abuse in Tanzania and Kenya. Child Abuse Negl. 2004;28(8):833-44. DOI: 10.1016/j. chiabu.2003.11.022

15. May-Chahal C, Cawson P. Measuring child maltreatement in the United Kingdom: A study of the prevalence of child abuse and neglect. Child Abuse Negl. 2005;29(9):969-84. DOI: 10.1016/j. chiabu.2004.05.009

16. Paveza GJ. Risk factors in father-daughter child sexual abuse. I Interpers Violence. 1988;3(3):290-306. DOI: $10.1177 / 088626088003003003$

17. Ramos-Lira L, Saldívar-Hernández G, MedinaMora ME, Rojas-Guiot E, Villatoro-Velázquez J. Prevalencia de abuso sexual en estudiantes y su relación con el consumo de drogas. Salud Publica Mexico. 1998;40(3):221-33. DOI: 10.1590/S003636341998000300002

18. Renner LM, Slack KS. Intimate partner violence and child maltreatment: Understanding intraand intergenerational connections. Child Abuse Negl. 2006;30(6):599-617. DOI: 10.1016/j. chiabu.2005.12.005

19. Russell DE. The incidence and prevalence of intrafamilial and extrafamilial sexual abuse of female children. Child Abuse Negl. 1983;7(2):133-46. DOI: 10.1016/0145-2134(83)90065-0

20. Silverman AB, Reinherz HZ, Giaconia RM. The long-term sequelae of child and adolescent abuse: a longitudinal community study. Child Abuse Negl. 1996;20(8):709-23. DOI: 10.1016/01452134(96)00059-2

21. Steel JL, Herlitz CA. The association between childhood and adolescent sexual abuse and proxies for sexual risk behavior: A random sample of the general population of Sweden. Child Abuse Negl. 2005;29(10):1141-53. DOI: 10.1016/j.chiabu.2004.10.015

22. Strauss MA, Gelles RJ. Societal change and change in family violence from 1975 to 1985 as revealed by two national surveys. J Marriage Fam. 1986;48(3):465-79. DOI: $10.2307 / 352033$

23. Summit RC. The child sexual abuse accommodation syndrome. Child Abuse Negl. 1983;7(2):177-93. DOI: 10.1016/0145-2134(83)90070-4

24. Thompson KM, Crosby RD, Wonderlich SA, Mitchell JE, Redlin J, Demuth G, et al. Psychopathology and sexual trauma in childhood and adulthood. I Trauma Stress. 2003;16(1):35-8. DOI: 10.1023/A:1022007327077

25. Wyatt GE. The sexual abuse of Afro-American and white-American women in childhood. Child Abuse Negl. 1985;9(4):507-19. DOI: 10.1016/01452134(85)90060-2

Pesquisa financiada por el Consejo Nacional de Ciencia y Tecnología de México (Proceso nº 37207-M). 